ROMA1/1425/06

OU-HET-556/2006

\title{
Effects of Bulk Mass in Gauge-Higgs Unification
}

\author{
Nobuhito $\operatorname{Maru}^{(a)}{ }^{1}$ and Kazunori Takenaga ${ }^{(b)} 2$ \\ (a) Dipartimento di Fisica, Università di Roma "La Sapienza" \\ and INFN, Sezione di Roma, P.le Aldo Moro, I-00185 Roma,Italy \\ (b) Department of Physics, Osaka University, Toyonaka, Osaka 560-0043, Japan
}

\begin{abstract}
We study effects of bulk mass on electroweak symmetry breaking and Higgs mass in the scenario of five dimensional $S U(3)$ gauge-Higgs unification defined on $M^{4} \times$ $S^{1} / Z_{2}$. The asymptotic form of effective potential for the Higgs field is obtained, from which a transparent and useful expression for the Higgs mass is found. The small vacuum expectation values (VEV) for Higgs field can be realized by choosing bulk mass parameters approriately for a fixed set of matter content. The bulk mass for periodic fermion field, in general, has effects to make the Higgs mass less heavy. On the other hand, the bulk mass for antiperiodic field does not directly affect the Higgs mass, but it contributes to increase or decrease the Higgs mass, depending on how small the VEV is induced due to the antiperiodic field. We give numerical examples to confirm these effects, in which the role of the bulk mass is also definitely clear.
\end{abstract}

\footnotetext{
${ }^{1}$ E-mail: Nobuhito.Maru@roma1.infn.it (N.Maru)

${ }^{2}$ E-mail: takenaga@tuhep.phys.tohoku.ac.jp (K.Takenaga)

Present address : Department of Physics, Tohoku University, Sendai, 980-8578 Japan.
} 


\section{Introduction}

The idea of gauge-Higgs unification, originally proposed by Manton [1] and Fairlie [2], is one of the attractive ideas for resolving the (little) gauge hierarchy problem [3]. In the gauge-Higgs unification, the Higgs field is unified as extra components in higher dimensional gauge field and is related to the Wilson line phases along the compactified space direction. The electroweak symmetry is broken through the dynamics of the Wilson line phases (Hosotani mechanism [4), and the Higgs field becomes massive, though it is massless at the tree-level, which is obtained by the effective potential induced by radiative corrections. The gauge-Higgs unification, including the dynamics of the Wilson line phases, has been studied from various points of view in the past [5, 6, 17.

The potential for the Higgs field generated at quantum level has a non local form, reflecting the nonlocal nature of the Wilson line phases [8], so that it is free from ultraviolet effects. That is why the mechanism can provide an alternative solution to the gauge hierarchy problem. Moreover, the Higgs sector in the gauge-Higgs unification has no free parameters. Once we fix matter content, the Higgs mass is calculable. The size of the Higgs mass, generally speaking, is small compared with the gauge boson mass because it is generated by the radiative correction, which is similar to the Coleman-Weinberg mechanism 9]. In order for the scenario to be valid, one needs the heavy Higgs mass consistent with the experimental lower bound.

For the heavy Higgs mass, it has been known that there are two points that have to be satisfied. The first point is that the vacuum expectation values (VEV) for the Higgs field must be small enough, and the second point is that one needs the matter belonging to the higher dimensional representation under the gauge group ${ }^{3}$ [10, 11]. The first point is realized by choosing an appropriate matter content in such a way that the coefficient of the negative mass term of the induced Higgs potential is very small [10].

Bulk mass plays an important role when we discuss the gauge symmetry breaking patterns through the dynamics of the Wilson line phases [12, 13] and fermion mass spectrum [14, 11] in the gauge-Higgs unification. The bulk mass is a necessary ingredient for model building based on the gauge-Higgs unification even though, as a result, it introduces free parameters in the Higgs sector. Therefore, it is important to understand the role and effect of the bulk mass in the gauge-Higgs unification ${ }^{4}$.

In this paper we study the effect of the bulk mass in nonsupersymmetric five dimensional $S U(3)$ gauge-Higgs unification theory, where an extra spatial coordinate is compactified on an orbifold $S^{1} / Z_{2}$. As is well known, the fermion bulk mass term in five dimensions is parity odd under the reflection of the coordinate of the extra dimension.

\footnotetext{
${ }^{3}$ It has been also reported that effects of the breakdown of the Lorentz invariance also enhance the Higgs mass [15].

${ }^{4}$ The role of the bulk mass is also studied from a point of view of low-energy effective theory of the gauge-Higgs unification [7]
} 
In order to have the parity even bulk mass term, we introduce a pair of the fields, each satisfying periodic or antiperiodic boundary condition in this paper. The effect of the periodic field in the gauge-Higgs unification is different from that of the antiperiodic field. We give an aymptotic form of the effective potential, from which the effect is definitely clear and, also from which a transparent and useful expression for the Higgs mass is found.

It is possible to have the small VEV for the Higgs field by choosing the bulk mass parameters appropriately for a fixed matter content. Namely, the bulk mass for the antiperiodic field is important to control the magnitude of the VEV. Since the effective potential depends on the bulk mass parameters, the Higgs mass also depends on them. The size of the Higgs mass is mainly controlled by the logarithmic factor arising only from the periodic fields. The bulk mass for the periodic field, in general, has effects to make the Higgs mass less heavy. In order to enhance the Higgs mass, one needs smaller VEV for moderate size of the bulk mass. On the other hand, the bulk mass itself for the antiperiodic field has tiny effects on the Higgs mass, but the field has a crucial role to make the VEV large (small), which accordingly can increases (decreases) the Higgs mass. We will present numerical examples, in which the effect of the bulk mass is definitely clear.

In the next section we present our model and study the electroweak symmetry breaking in the gauge-Higgs unification, paying attention to the size of the VEV for the Higgs field and the Higgs mass. In section 3, some numerical examples are given. Section 4 is devoted to conclusions and discussions. The derivation of asymptotic form of the potential is breifly summarized in an appendix.

\section{Bulk mass parameter and effective potential}

We consider nonsupersymmetric gauge theory on $M^{4} \times S^{1} / Z_{2}$ with the gauge group $S U(3)$, where $M^{4}$ is the four dimensional Minkowski space-time and $S^{1} / Z_{2}$ is an orbifold ${ }^{5}$. One needs to specify boundary conditions of fields for the $S^{1}$ direction and the two fixed points located at $y=0, \pi R$. These boundary conditions are defined by the two matrices, $P_{0}=P_{1}=$ diag. $(-1,-1,1)$, from which the original gauge symmetry is broken down to $S U(2) \times U(1)$ [16. And the Higgs field is embedded in the zero modes of the extra component of the gauge field $A_{y}$ as

$$
A_{y}^{(0)}=\frac{1}{2}\left(\begin{array}{rl} 
& A_{y}^{4}-i A_{y}^{5} \\
& A_{y}^{6}-i A_{y}^{7}
\end{array}\right) \quad \text { where } \Phi \equiv \sqrt{2 \pi R}\left(\begin{array}{c}
A_{y}^{4}-i A_{y}^{5} \\
A_{y}^{6}-i A_{y}^{7}
\end{array}\right) \text {, }
$$

where $R$ is a radius of the $S^{1}$. $\Phi$ transforms as a doublet under the $S U(2)$.

\footnotetext{
${ }^{5}$ The gauge-Higgs unification in supersymmetric gauge theory with bulk mass is also studied in [10].
} 
The VEV of the Higgs field is parametrized by

$$
\left\langle A_{y}^{(0)}\right\rangle=\frac{a}{g_{4} R} \frac{\lambda^{6}}{2} \equiv A_{y}^{6(0)} \frac{\lambda^{6}}{2} .
$$

Here, $g_{4}$ stands for the four dimensional gauge coupling defined by the original five dimensional coupling $g_{5}$ by $g_{4} \equiv g_{5} / \sqrt{2 \pi R}$, and $a$ is a real constant. The VEV $a$ is related to the Wilson line phase and determines the patterns of the gauge symmetry breaking,

$$
\begin{aligned}
W & =\mathcal{P} \exp \left(i g \oint_{S^{1}} d y\left\langle A_{y}\right\rangle\right)=\left(\begin{array}{ccc}
1 & 0 & 0 \\
0 & \cos \left(\pi a_{0}\right) & i \sin \left(\pi a_{0}\right) \\
0 & i \sin \left(\pi a_{0}\right) & \cos \left(\pi a_{0}\right)
\end{array}\right) \quad\left(a_{0} \bmod 2\right) \\
& =\left\{\begin{array}{lll}
S U(2) \times U(1) & \text { for } & a_{0}=0, \\
U(1)^{\prime} \times U(1) & \text { for } & a_{0}=1, \\
U(1)_{e m} & \text { for } & \text { otherwise. }
\end{array}\right.
\end{aligned}
$$

$a_{0}$ represents the value of $a$ at the minimum of the potential. In order to determine the values of the Wilson line phase $a$, one usually evaluates the effective potential for the phase and minimizes it. Then, the Higgs mass is calculated by the second derivative of the effective potential at the minimum. Since $a_{0}$ is related to weak gauge boson masses whose size is given by $a_{0} / R$, if one requires $1 / R \sim$ a few TeV, then, $a_{0}$ must be of order of $O\left(10^{-2}\right)$. This is a strong constraint that should be satisfied in gauge-Higgs unification.

We consider the matter field with bulk mass term. The bulk mass term for fermion in five dimensions is odd under the parity transformation, $y \rightarrow-y(\pi R-y \rightarrow \pi R+y)$. We need parity even mass term for the consistency of the $Z_{2}$ orbifolding. The parity even mass term is introduced by the coordinate dependent mass term such as, for example, $M(-y)=-M(y)$, where $M(y)=\epsilon(y) M(\epsilon(y)$ is the step function). The other way for obtaining the parity even mass term is to introduce a pair of the fields, $\psi_{+}$and $\psi_{-}$whose parity is different to each other, $\psi_{ \pm}(-y)= \pm \psi_{ \pm}(y)$. Then, a parity even mass term is constructed like $M \bar{\psi}_{+} \psi_{-}$. In this paper we follow this case.

Let us suppose that $\psi^{( \pm)}$and $\tilde{\psi}^{( \pm)}$belong to the fundamental representation under the $S U(3)$ gauge group and satisfy the following boundary conditions ${ }^{6}$,

$$
\begin{aligned}
& \text { type I ; }\left\{\begin{array} { l } 
{ \psi ^ { ( + ) } ( - y ) = P _ { 0 } i \Gamma ^ { y } \psi ^ { ( + ) } ( y ) } \\
{ \psi ^ { ( - ) } ( - y ) = - P _ { 0 } i \Gamma ^ { y } \psi ^ { ( - ) } ( y ) , }
\end{array} \quad \left\{\begin{array}{l}
\psi^{(+)}(\pi R-y)=P_{1} i \Gamma^{y} \psi^{(+)}(\pi R+y) \\
\psi^{(-)}(\pi R-y)=-P_{1} i \Gamma^{y} \psi^{(-)}(\pi R+y) .
\end{array}\right.\right. \\
& \text { type II ; }\left\{\begin{array} { l } 
{ \tilde { \psi } ^ { ( + ) } ( - y ) = P _ { 0 } i \Gamma ^ { y } \tilde { \psi } ^ { ( + ) } ( y ) } \\
{ \tilde { \psi } ^ { ( - ) } ( - y ) = - P _ { 0 } i \Gamma ^ { y } \tilde { \psi } ^ { ( - ) } ( y ) , }
\end{array} \left\{\begin{array}{l}
\tilde{\psi}^{(+)}(\pi R-y)=-P_{1} i \Gamma^{y} \tilde{\psi}^{(+)}(\pi R+y) \\
\tilde{\psi}^{(-)}(\pi R-y)=P_{1} i \Gamma^{y} \tilde{\psi}^{(-)}(\pi R+y) .
\end{array}\right.\right.
\end{aligned}
$$

We can also consider the boundary conditions where the overall sign is different from the above boundary conditions, but these contributions to the effective potential are the same as those defined in Eqs. (44) and (5). It is easy to see that a pair $\left(\psi^{(+)}, \psi^{(-)}\right)\left(\left(\tilde{\psi}^{(+)}, \tilde{\psi}^{(-)}\right)\right.$

\footnotetext{
${ }^{6}$ Notation used in this section is the same as those in [10].
} 
can have the parity even and gauge invariant mass term, $M \bar{\psi}^{( \pm)} \psi^{(\mp)}\left(M \overline{\tilde{\psi}}^{( \pm)} \tilde{\psi}^{(\mp)}\right)$. Taking into account the boundary conditions (4) and (15), $\psi^{( \pm)}$is expanded in terms of $\cos \left(\frac{n}{R} y\right)$ and $\sin \left(\frac{n}{R} y\right)$, while $\tilde{\psi}^{( \pm)}$is expanded in terms of $\cos \left(\frac{\left(n+\frac{1}{2}\right) y}{R}\right)$ and $\sin \left(\frac{\left(n+\frac{1}{2}\right) y}{R}\right)$. We call the field with the expansion $\cos \left(\frac{n}{R} y\right)\left(\sin \left(\frac{n}{R} y\right)\right)$ periodic field, while that with the expansion $\cos \left(\frac{\left(n+\frac{1}{2}\right) y}{R}\right)\left(\sin \left(\frac{\left(n+\frac{1}{2}\right) y}{R}\right)\right)$ antiperiodic field.

The contributions to the effective potential from the fermions $\psi^{(+)}$and $\psi^{(-)}$is given by

$$
V_{e f f}^{I}=-2^{\left[\frac{5}{2}\right]} N_{I}^{\text {pair }}(1+1) \frac{1}{L} \frac{1}{2} \sum_{n=-\infty}^{\infty} \int \frac{d^{4} p_{E}}{(2 \pi)^{4}} \ln \left[p_{E}^{2}+\left(\frac{n+\frac{a}{2}}{R}\right)^{2}+M^{2}\right],
$$

where $N_{I}^{\text {pair }}$ stands for the number of the pair $\left(\psi^{(+)}, \psi^{(-)}\right)$. The overall minus sign comes for the Fermi statistics. $p_{E}$ denotes Euclidean momentum. Likewise, we obtain from the pair $\left(\tilde{\psi}^{(+)}, \tilde{\psi}^{(-)}\right)$that

$$
V_{e f f}^{I I}=-2^{\left[\frac{5}{2}\right]} N_{I I}^{\text {pair }}(1+1) \frac{1}{L} \frac{1}{2} \sum_{n=-\infty}^{\infty} \int \frac{d^{4} p_{E}}{(2 \pi)^{4}} \ln \left[p_{E}^{2}+\left(\frac{n+\frac{a}{2}-\frac{1}{2}}{R}\right)^{2}+M^{2}\right] \text {, }
$$

where $N_{I}^{\text {pair }}$ stands for the number of the pair $\left(\tilde{\psi}^{(+)}, \tilde{\psi}^{(-)}\right)$. According to the usual prescription [19, it is easy to evaluate Eqs. (6) and (17) as

$$
\begin{aligned}
& \frac{1}{2} \frac{1}{L} \sum_{n=-\infty}^{\infty} \int \frac{d^{4} p_{E}}{(2 \pi)^{4}} \ln \left[p_{E}^{2}+\left(\frac{n+Q a-\frac{\delta}{2}}{R}\right)^{2}+M^{2}\right] \\
= & -\frac{2}{(2 \pi)^{\frac{5}{2}}} \sum_{n=1}^{\infty}\left(\frac{M}{n L}\right)^{\frac{5}{2}} K_{\frac{5}{2}}(n L M) \cos \left[2 \pi n\left(Q a-\frac{\delta}{2}\right)\right] \\
= & -\frac{3}{4 \pi^{2}} \frac{1}{L^{5}} \sum_{n=1}^{\infty} \frac{1}{n^{5}}\left(1+n z+\frac{n^{2} z^{2}}{3}\right) \mathrm{e}^{-n z} \cos \left[2 \pi n\left(Q a-\frac{\delta}{2}\right)\right],
\end{aligned}
$$

where we have ignored the irrelevant constant. $\delta$ takes $0(1)$ for (anti)periodic field. We have defined the dimensionless variable $z \equiv M L$ and also used the fact that the modified Bessel function $K_{\nu}(x)$ can be rewritten as

$$
K_{\frac{5}{2}}(x)=3\left(\frac{\pi}{2 x^{5}}\right)^{\frac{1}{2}}\left(1+x+\frac{x^{2}}{3}\right) \mathrm{e}^{-x} .
$$

Then, the effective potential from the matter is summarized into the form as

$$
V_{e f f}(a, z, \delta)=(-1)^{F+1} N_{\text {deg }}\left(2 N_{\text {pair }}\right) \frac{3}{4 \pi^{2} L^{5}} f(Q a, z, \delta),
$$

where

$$
f(Q a, z, \delta)=\sum_{n=1}^{\infty} \frac{1}{n^{5}}\left(1+n z+\frac{n^{2} z^{2}}{3}\right) \mathrm{e}^{-n z} \cos \left[2 \pi n\left(Q a-\frac{\delta}{2}\right)\right] .
$$

$N_{\text {deg }}$ is the on-shell degrees of freedom of the concerned field, and $F$ stands for the fermion number. Let us note that the factor 2 in $2 N_{\text {pair }}$ comes from $\psi^{(+)}$and $\psi^{(-)}$in the pair $\left(\psi^{(+)}, \psi^{(-)}\right)$. 
The "charge" $Q$ is just the magnitude of the $S U(2)$ spin $j$. Any representation of the $S U(3)$ is decomposed in terms of the irreducible representation of $S U(2)$. The fundamental representation of $S U(3)$, for example, is decomposed by an $S U(2)$ doublet and a singlet, so that $Q=j=1 / 2$ from the doublet contribution, and the singlet contribution does not depend on the order parameter $a$.

Following the previous work done by the authors [18, we introduce the matter fields whose flavor numbers are specified by

$$
\left(N_{a d j}^{I}, N_{f d}^{I}, N_{a d j}^{(+) s}, N_{f d}^{(+) s} ; N_{a d j}^{I I}, N_{f d}^{I I}, N_{a d j}^{(-) s}, N_{f d}^{(-) s}\right) .
$$

Here, we have also introduced the scalar field with the $\eta=-1$ parity ${ }^{7}$, which is essentially the same as the antiperiodic field. The mode expansion of the scalar field with $\eta=+1(-1)$ is the same as those of the fermions with type I (II) boundary conditions. Then, the effective potential for these flavor number of fields (12) is given by

$$
\begin{aligned}
\bar{V}_{e f f}(a)=\frac{V_{e f f}}{3 / 4 \pi^{2} L^{5}} & =-3(f(2 a, 0,0)+2 f(a, 0,0)) \\
& +4\left(2 N_{a d j}^{I}\right)\left(f\left(2 a, z_{a d j}^{(+)}, 0\right)+2 f\left(a, z_{a d j}^{(+)}, 0\right)\right) \\
& +4\left(2 N_{a d j}^{I I}\right)\left(f\left(2 a, z_{a d j}^{(-)}, 1\right)+2 f\left(a, z_{a d j}^{(-)}, 1\right)\right) \\
& +4\left(2 N_{f d}^{I}\right) f\left(a, z_{f d}^{(+)}, 0\right)+4\left(2 N_{f d}^{I I}\right) f\left(a, z_{f d}^{(-)}, 1\right) \\
& -d N_{a d j}^{(+) s}\left(f\left(2 a, z_{a d j}^{(+) s}, 0\right)+2 f\left(a, z_{a d j}^{(+) s}, 0\right)\right) \\
& -d N_{a d j}^{(-) s}\left(f\left(2 a, z_{a d j}^{(-) s}, 1\right)+2 f\left(a, z_{a d j}^{(-) s}, 1\right)\right) \\
& -\left(2 N_{f d}^{(+) s}\right) f\left(a, z_{f d}^{(+) s}, 0\right)-\left(2 N_{f d}^{(-) s}\right) f\left(a, z_{f d}^{(-) s}, 1\right) .
\end{aligned}
$$

The first line in Eq.(13) comes from the gauge sector. The factor $d$ in the adjoint scalar contributions takes $1(2)$ for the real (complex) field.

In order to see the role of the bulk mass clearly, let us study the limit $z \ll 1$ and obtain the asymptotic form of $f(Q a, z, \delta)$. Taking also into account the smallness of the VEV for the Higgs field $a$, one can utilize the formulae given in the appendix. Then, we find that

$$
\begin{aligned}
f(x, z, 0) & \simeq \zeta(5)-\frac{\zeta(3)}{6} z^{2}+\frac{z^{4}}{32}-\frac{\zeta(3)}{2} x^{2}+\frac{1}{48}\left(7 x^{2} z^{2}+\frac{25}{6} x^{4}\right) \\
& -\frac{1}{48}\left(x^{2}+z^{2}\right)^{2} \ln \left(x^{2}+z^{2}\right), \\
f(x, z, 1) & \simeq-\frac{15}{16} \zeta(5)+\frac{\zeta(3)}{8} z^{2}+\frac{3}{8} \zeta(3) x^{2}-\frac{\ln 2}{24}\left(x^{2}+z^{2}\right)^{2},
\end{aligned}
$$

where $x \equiv 2 \pi Q a$, and we have ignored higher order terms. The equation (14) is the same as that obtained in [7].

\footnotetext{
${ }^{7}$ Definition for the $\eta$ parity was given in 20
} 
Let us apply the formulae (14) and (15) to the effective potential (13). We obtain, apart from irrelevant constant, that

$$
\begin{aligned}
\bar{V}_{e f f} & \simeq-\frac{\pi^{2} B}{2} a^{2}+\frac{\pi^{4} C}{48} a^{4}+\frac{9 \pi^{4}}{8} a^{4} \ln (\pi a)^{2} \\
& -\frac{1}{48}\left(4\left(2 N_{a d j}^{I}\right)\left[L\left(2 a, z_{a d j}^{(+)}\right)+2 L\left(a, z_{a d j}^{(+)}\right)\right]+4\left(2 N_{f d}^{I}\right) L\left(a, z_{f d}^{(+)}\right)\right. \\
& \left.-d N_{a d j}^{(+) s}\left[L\left(2 a, z_{a d j}^{(+) s}\right)+2 L\left(a, z_{a d j}^{(+) s}\right)\right]-2 N_{f d}^{(+) s} L\left(a, z_{f d}^{(+)}\right)\right),
\end{aligned}
$$

where

$$
L(a, z) \equiv\left((\pi a)^{2}+z^{2}\right)^{2} \ln \left[(\pi a)^{2}+z^{2}\right] .
$$

The coefficients $B, C$ are given by

$$
\begin{aligned}
B & \equiv \zeta(3)\left[48 N_{a d j}^{I}+8 N_{f d}^{I}+\frac{3}{4}\left(6 d N_{a d j}^{(-) s}+2 N_{f d}^{(-) s}\right)\right. \\
& \left.-\frac{3}{4}\left(48 N_{a d j}^{I I}+8 N_{f d}^{I I}\right)-\left(6 d N_{a d j}^{(+) s}+2 N_{f d}^{(+) s}+18\right)\right] \\
& -\frac{7}{24}\left(48 N_{a d j}^{I} z_{a d j}^{(+) 2}+8 N_{f d}^{I} z_{f d}^{(+) 2}\right)+\frac{\ln 2}{6}\left(48 N_{a d j}^{I I} z_{a d j}^{(-) 2}+8 N_{f d}^{I I} z_{f d}^{(-) 2}\right) \\
& +\frac{7}{24}\left(6 d N_{a d j}^{(+) s} z_{a d j}^{(+) s 2}+2 N_{f d}^{(+) s} z_{f d}^{(+) s 2}\right)-\frac{\ln 2}{6}\left(6 d N_{a d j}^{(-) s} z_{a d j}^{(-) s 2}+2 N_{f d}^{(-) s} z_{f d}^{(-) s 2}\right), \\
C & \equiv \frac{25}{6}\left(72\left(2 N_{a d j}^{I}\right)+4\left(2 N_{f d}^{I}\right)-18 d N_{a d j}^{(+) s}-2 N_{f d}^{(+) s}-54\right) \\
& +2 \ln 2\left(18 d N_{a d j}^{(-) s}+2 N_{f d}^{(-) s}+54-72\left(2 N_{a d j}^{I I}\right)-4\left(2 N_{f d}^{I I}\right)\right) .
\end{aligned}
$$

If we do not introduce the bulk mass at all, in order to obtain the small VEV for the Higgs field, we need to choose the matter content in such a way that the coefficient $B$ is almost cancelled [10, that is, the cancellation between the first and the second lines in Eq.(18) is required. Here, thanks to the bulk mass parameters, we can make the coefficient $B$ small by choosing the parameters appropriately even for a fixed matter content.

In general, we see that the massive bulk fermion with $\delta=0$ has effects to restore the gauge symmetry because of the positive mass term $7 z^{2} x^{2} / 48$ in Eq.(14), while from Eq. (15), the massive bulk fermion with $\delta=1$ has effects to break the gauge symmetry due to the negative mass term $(\ln 2 / 12) z^{2} x^{2}$. Let us also note that the boson with $\eta=+(-)$ parity has the same role as the fermion with $\delta=1(0)$.

The Higgs mass is obtained from the second derivative of the effective potential at the minimum. The asymptotic expression for the Higgs mass is given, using (16) and the relation $a_{0} / g_{4} R=v[21$, by

$$
m_{H}^{2}=\left.g_{4}^{4} \frac{3}{64 \pi^{6}}\left(\frac{v}{a_{0}}\right)^{2} \frac{\partial^{2} \bar{V}_{e f f}}{\partial a^{2}}\right|_{a=a_{0}},
$$

where

$$
\frac{\partial^{2} \bar{V}_{e f f}}{\partial a^{2}}=8 \pi^{4} a_{0}^{2}\left[\frac{-4\left(2 N_{a d j}^{I}\right)}{48}\left(2^{4} H\left(2 a, z_{a d j}^{(+)}\right)+2 \times 1^{4} H\left(a, z_{a d j}^{(+)}\right)\right)\right.
$$




$$
\begin{aligned}
& -\frac{4\left(2 N_{f d}^{I}\right)}{48} H\left(a, z_{f d}^{(+)}\right)+\frac{9}{8}\left(\ln \left(\pi^{2} a^{2}\right)+\frac{3}{2}\right)+\frac{C}{48} \\
& +\frac{d N_{a d j}^{(+) s}}{48}\left(2^{4} H\left(2 a, z_{a d j}^{(+) s}\right)+2 \times 1^{4} H\left(a, z_{a d j}^{(+) s}\right)\right) \\
& \left.+\frac{2 N_{f d}^{(+) s}}{48} 1^{4} H\left(a, z_{f d}^{(+) s}\right)\right],
\end{aligned}
$$

where

$$
H(a, z)=\ln \left[(\pi a)^{2}+z^{2}\right]+\frac{3}{2}
$$

We observe that the Higgs mass depends on the magnitude of the $S U(2)$ spin like $(2 Q)^{4}\left(=2^{4}, 1^{4}\right)$, in front of $H(a, z)$, so that the matter with the higher dimensional representation under the gauge group enhances the size of the Higgs mass [11, 10].

A remarkable feature is that the bulk mass itself of the field with $\delta=1$ does not affect the Higgs mass directly, but it contributes to increase or decrease the Higgs mass only through the magnitude of the VEV for the Higgs field. The bulk mass for the field with $\delta=1$ has a role of changing the magnitude. We will explicitly show such examples in the next section.

On the other hand, the bulk mass for the field with $\delta=0$ can have sizable effect on the Higgs mass. First of all, let us note that the large logarithmic factor due to the small VEV $a_{0} \ll 1$ comes from only the field with $\delta=0$, as seen from Eq.(21). In our numerical analyses given below, we do not consider the scalar fields with $\delta=0$, so that the Higgs mass is dominantly controlled by the fermion fields with $\delta=0$ (and the gauge field). However, the bulk mass for the field with $\delta=0$ modifies the argument of the logarithm, which implies that the bulk mass tends to decrease the size of the Higgs mass in general. Therefore, in order to avoid the light Higgs mass, one needs to have smaller values of the VEV $a_{0}$ for moderate size of the bulk mass, which overcomes the $z^{2}$-suppression in the argument of the logarithm.

\section{$3 \quad$ Numerical results}

In this section, let us show numerical results for the following three cases,

$$
\begin{aligned}
& \text { (A) }\left(N_{a d j}^{I}, N_{f d}^{I}, N_{a d j}^{(+) s}, N_{f d}^{(+) s} ; N_{a d j}^{I I}, N_{f d}^{I I}, N_{a d j}^{(-) s}, N_{f d}^{(-) s}\right)=(1,1,0,0 ; 1,1,1,0), \\
& \text { (B) }\left(N_{a d j}^{I}, N_{f d}^{I}, N_{a d j}^{(+) s}, N_{f d}^{(+) s} ; N_{a d j}^{I I}, N_{f d}^{I I}, N_{a d j}^{(-) s}, N_{f d}^{(-) s}\right)=(1,1,0,2 ; 1,1,2,0), \\
& \text { (C) }\left(N_{a d j}^{I}, N_{f d}^{I}, N_{a d j}^{(+) s}, N_{f d}^{(+) s} ; N_{a d j}^{I I}, N_{f d}^{I I}, N_{a d j}^{(-) s}, N_{f d}^{(-) s}\right)=(1,1,0,0 ; 1,1,0,3) .
\end{aligned}
$$


We first present the numerical results for the case $(A)$ in the table below ${ }^{8}$.

\begin{tabular}{|c|cccc|ccccccc|}
\hline & $z_{a d j}^{(+)}$ & $z_{f d}^{(+)}$ & $z_{a d j}^{(+) s}$ & $z_{f d}^{(+) s}$ & $z_{a d j}^{(-)}$ & $z_{f d}^{(-)}$ & $z_{a d j}^{(-) s}$ & $z_{f d}^{(-) s}$ & $\frac{1}{g_{4} R}$ & $a_{0}$ & $m_{H} / g_{4}^{2}$ \\
\hline \hline$(1)$ & 0 & 0 & - & - & 0 & 0 & 0 & - & 6.3 & 0.039 & 134.0 \\
\hline$(2)$ & 0.1 & 0.2 & - & - & 0 & 0 & 0 & - & 12.4 & 0.020 & 139.4 \\
\hline$(3)$ & 0 & 0 & - & - & 0 & 0 & 0.5 & - & 7.7 & 0.032 & 141.0 \\
\hline$(4)$ & 0.1 & 0.2 & - & - & 0 & 0 & 0.2 & - & 14.0 & 0.018 & 140.4 \\
\hline$(5)$ & 0.1 & 0.2 & - & - & 0.1 & 0 & 0 & - & 10.4 & 0.024 & 137.2 \\
\hline$(6)$ & 0.1 & 0.2 & - & - & 0 & 0.2 & 0 & - & 11.0 & 0.022 & 137.9 \\
\hline$(7)$ & 0.2 & 0.2 & - & - & 0.3 & 0.3 & 0 & - & 24.0 & 0.010 & 109.1 \\
\hline
\end{tabular}

In the table (and the subsequence tables), the Higgs mass is measured in GeV unit and the radius of the $S^{1}$ is in TeV unit. The case (1) corresponds to the massless bulk fields.

The equation (21) implies that the bulk mass $z_{a d j(f d)}^{(+)}$tends not to make the Higgs mass heavy. In order to have the heavy Higgs mass, the VEV $a_{0}$ for the Higgs field has to be smaller. The case (2) just shows that the Higgs mass is enhanced by the smaller $\mathrm{VEV}$ for the moderate size of the introduced bulk mass $z_{\text {adj }(f d)}^{(+)}$.

The cases (3) - (6) are examples showing the effect of the bulk mass for the field with $\delta=1$ on the Higgs mass. From Eq. (21), the field with $\delta=1$ does not affect the Higgs mass directly, but it contributes to increase or decrease the Higgs mass, depending on how small the VEV $a_{0}$ for the Higgs field is induced due to the bulk mass $z^{(-)}$. The cases (3) and (4) show that the bulk mass $z_{\text {adj }}^{(-)}$s makes the VEV small. As a result, the Higgs mass is enhanced compared with the corresponding cases with $z_{\text {adj }}^{(-) s}=0$. On the other hand, the cases (5) and (6) show that the bulk mass $z_{a d j(f d)}^{(-)}$decreases the Higgs mass because it does not induce the smaller VEV $a_{0}$ compared with the correspondeing cases with $z_{\text {adj }(f d)}^{(-)}=0$.

Although the very small VEV $a_{0}$ is realized in (7) by adjusting the bulk mass parameres, the effects of $\delta=0$ fermions with the large bulk masses dominate in the argument of the logarithm. That is why the Higgs mass is light in this case.

Let us next present the results for the case (B), which is summarized in the table,

\begin{tabular}{|c|cccc|ccccccc|}
\hline & $z_{a d j}^{(+)}$ & $z_{f d}^{(+)}$ & $z_{a d j}^{(+) s}$ & $z_{f d}^{(+) s}$ & $z_{a d j}^{(-)}$ & $z_{f d}^{(-)}$ & $z_{a d j}^{(-) s}$ & $z_{f d}^{(-) s}$ & $\frac{1}{g_{4} R}$ & $a_{0}$ & $m_{H} / g_{4}^{2}$ \\
\hline \hline$(1)$ & 0 & 0 & - & 0 & 0 & 0 & 0 & - & 4.0 & 0.062 & 117.4 \\
\hline$(2)$ & 0.2 & 0.2 & - & 0.2 & 0 & 0 & 0 & - & 10.4 & 0.024 & 118.6 \\
\hline$(3)$ & 0.2 & 0.1 & - & 0.2 & 0 & 0 & 0 & - & 7.7 & 0.032 & 120.0 \\
\hline$(4)$ & 0.2 & 0.25 & - & 0.2 & 0 & 0 & 0 & - & 15.5 & 0.016 & 115.0 \\
\hline$(5)$ & 0.2 & 0.3 & - & 0.5 & 0 & 0 & 0 & - & 7.5 & 0.033 & 118.2 \\
\hline
\end{tabular}

In these cases, the Higgs mass slightly small compared with the previous cases though the VEV $a_{0}$ is almost the same values. This is clear from the fact that the all the fields with

\footnotetext{
${ }^{8}$ The present model cannot produce the correct Weinberg angle, so that we take the four dimensional gauge coupling to be a free parameter, and it is assumed to be of order of $O(1)$.
} 
$\delta=0$ have the bulk mass, so that the logarithmic factor, which could make the Higgs mass heavy, cannot be large due to the increased argument in the logarithm.

Finally let us present the numerical results for the case $(\mathrm{C})$, which is given by

\begin{tabular}{|c|cccc|ccccccc|}
\hline & $z_{a d j}^{(+)}$ & $z_{f d}^{(+)}$ & $z_{a d j}^{(+) s}$ & $z_{f d}^{(+) s}$ & $z_{a d j}^{(-)}$ & $z_{f d}^{(-)}$ & $z_{a d j}^{(-) s}$ & $z_{f d}^{(-) s}$ & $\frac{1}{g_{4} R}$ & $a_{0}$ & $m_{H} / g_{4}^{2}$ \\
\hline \hline$(1)$ & 0 & 0 & - & - & 0 & 0 & - & 0 & 6.1 & 0.040 & 130.2 \\
\hline$(2)$ & 0.1 & 0 & - & - & 0 & 0 & - & 0 & 8.2 & 0.030 & 132.6 \\
\hline$(3)$ & 0.1 & 0.1 & - & - & 0 & 0 & - & 0 & 8.9 & 0.028 & 133.2 \\
\hline$(4)$ & 0.1 & 0.2 & - & - & 0 & 0 & - & 0 & 12.2 & 0.020 & 136.3 \\
\hline$(5)$ & 0 & 0.4 & - & - & 0 & 0 & - & 0 & 19.2 & 0.013 & 161.0 \\
\hline$(6)$ & 0.09 & 0.3 & - & - & 0 & 0 & - & 0 & 28.4 & 0.0087 & 139.7 \\
\hline$(7)$ & 0.08 & 0.3 & - & - & 0 & 0 & - & 0 & 17.3 & 0.014 & 143.2 \\
\hline
\end{tabular}

The case (6) (also the cases (5), (7) and (7) for the case (A)) is an example that the bulk mass is finely tuned for the coefficient of the quadratic terms for $a^{2}$ to yield the very small values of the VEV $a_{0}$. In this case, one needs to take care whether or not the very small values of $a_{0}$ is reliable result within the framework of perturbation theory. Since the number of order $O\left(10^{-3}\right)$ is smaller or at least comparable to the loop factor, one needs to check the stability of obtained result against higher loop corrections.

In the case (5), if we take the color factor 3 into account ${ }^{9}$, the Higgs mass $m_{H} \simeq 120$ $\mathrm{GeV}$ is consistent with the experimental lower bound even if we take the four dimensional gauge coupling to be the $S U(2)$ gauge coupling. The rough estimation tells us that in order for $\sqrt{3} g_{S U(2)}^{2} Q^{2}$, which is equivalent to the square of the effective coupling $g_{\text {eff }}^{2}$, to be $O(1)$, it is enough to introduce the matter with $Q=j=3 / 2$, that is, 10 of $S U(3)$.

\section{Conclusions and discussions}

We have considered the five dimensional $S U(3)$ gauge-Higgs unification theory defined on $M^{4} \times S^{1} / Z_{2}$. Its matter content includes a pair of the fields satisfying the periodic and the antiperiodic boundary conditions. Then, the parity even mass terms composed of these fields are introduced. We have studied the effect of the bulk mass in the scenario of the gauge-Higgs unification, in which the bulk mass is important when we discuss the realistic fermion mass spectrum.

In particular, we have investigated the effect of the bulk mass on the vacuum expectation values for the Higgs field and the size of the Higgs mass. The analyses have been performed using the transparent and useful expressions (16) and (21), which helps us to understand the effect of the bulk mass clearer.

We have found that the VEV for the Higss field $a_{0}$ can be small, which is necessary for the scenario of the gauge-Higgs unification, due to the bulk mass parameters for a fixed

\footnotetext{
${ }^{9}$ In this case, we start with the $S U(3)_{c} \times S U(3)$ group and set the orbifolding boundary condition for the $S U(3)_{c}$ is trivial.
} 
set of matter content. The effect of the bulk mass on the VEV is different, depending on the periodicity of the field. We have found in our numerical analyses that the bulk mass $z_{a d j}^{(-) s}\left(z_{a d j(f d)}^{(-)}\right)$is likely to make the VEV smaller (larger).

The size of the Higgs mass is also affected by the bulk mass parameters. The dominant contribution to the Higgs mass comes from only the field with $\delta=0$. The bulk mass for the field with $\delta=0$, however, tends to make the Higgs mass less heavy because it increases the argument of the logarithm in Eq.(21). One needs smaller values for the VEV in order to obtain the heavy Higgs mass for the moderate size of the bulk mass. On the other hand, the direct effect of the bulk mass for the field with $\delta=1$ on the Higgs mass is tiny, but it has a role to make the VEV large or small. We have especially observed that the Higgs mass is enhanced (decreased) by the bulk mass $z_{\text {adj }}^{(-) s}\left(z_{\text {adj }(f d)}^{(-)}\right)$.

For some cases that we have numerically studied, the VEV $a_{0}$ becomes the order of $O\left(10^{-3}\right)$. In this case, one needs to take care the stability of obtained result against higher order loop corrections to the effective potential since the order is almost the same as the loop factor. In this respect, as in the case with the matter in the higher dimensional representation under the gauge group for which the radiative corrections is sizable [11, we need to investigate the two loop effects to the effective potential when we have such the small VEV.

We have clarified in this paper that the bulk mass plays crucial roles to have the correct electroweak symmetry breaking (small VEV $a_{0}$ ) and the size of the Higgs mass. In connection with the electroweak phase transition at finite temperature through the dynamics of the Wilson line phases [22, 18, it is very interesting to study the effect of the bulk mass on the order of the phase transition. In particular, it is important to understand how the bulk mass affects the first order phase transition, which is necessary for the scenario of the electroweak baryogenesis. This will be reported soon [17].

\section{Acknowledgements}

N.M. is supported by I.N.F.N., sezione di Roma. K.T. is supported by the 21st Century COE Program at Osaka University and would like to thank Professor Y. Hosotani for fruitful discussions. He would also like to thank Dr. T. Yamashita for useful comments on the gauge-Higgs unification. K.T. would also like to thank the Yukawa Institute for Theoretical Physics for warm hospitality, where a part of this work has done.

\section{Appendix}

In this appendix, we briefly explain how the expressions (14) and (15) are derived. The 
function $f(Q a, z, \delta)$ is also written as

$$
\begin{aligned}
f(Q a, z, \delta) & =\operatorname{Re} \operatorname{Li}_{5}\left(\mathrm{e}^{-z+2 \pi i\left(Q a-\frac{\delta}{2}\right)}\right)+z \operatorname{Re}^{2} \operatorname{Li}_{4}\left(\mathrm{e}^{-z+2 \pi i\left(Q a-\frac{\delta}{2}\right)}\right) \\
& +\frac{z^{2}}{3} \operatorname{Re} \operatorname{Li}_{3}\left(\mathrm{e}^{-z+2 \pi i\left(Q a-\frac{\delta}{2}\right)}\right),
\end{aligned}
$$

where the polylogarithm function is defined by

$$
\operatorname{Li}_{D}\left(\mathrm{e}^{-z}\right)=\sum_{n=1}^{\infty} \frac{\mathrm{e}^{-n z}}{n^{D}} .
$$

The asymptotic form for $z \ll 1$ is given by 23 ]

$$
\begin{aligned}
\operatorname{Li}_{D}\left(\mathrm{e}^{-z}\right) & \simeq \sum_{n=0(n \neq D-1)}^{\infty}(-1)^{n} \frac{z^{n}}{n !} \zeta(D-n) \\
& +(-1)^{D} \frac{z^{D-1}}{(D-1) !}\left(\ln z-\left(1+\frac{1}{2}+\frac{1}{3}+\cdots+\frac{1}{D-1}\right)\right), \\
\operatorname{Li}_{D}\left(-\mathrm{e}^{-z}\right) & \simeq \sum_{n=0}^{D-2}(-)^{n+1} \frac{z^{n}}{n !} \eta(D-n)+(-)^{D} \frac{z^{D-1}}{(D-1) !} \ln 2+(-)^{D+1} \frac{1}{2} \frac{z^{D}}{D !} \\
& +(-)^{D} \sum_{m=1}^{\infty} \frac{z^{D+2 m-1}}{(D+2 m-1) !} \eta(1-2 m),
\end{aligned}
$$

where $\eta(s) \equiv\left(1-2^{1-s}\right) \zeta(s)$. In order to obtain the expressions (14) and (15), we first expand the $\cos (n a)$ with respect to $a$ since $a$ is small. In addition to the formulae given above, using the useful expressions

$$
\begin{aligned}
\sum_{n=1}^{\infty} \frac{1}{n} \mathrm{e}^{-n z} & =\ln \left(1-\mathrm{e}^{-z}\right)^{-1} \simeq-\ln (z)+\frac{z}{2}-\frac{z^{2}}{24}+\frac{z^{4}}{2880}-\frac{z^{6}}{181440}+O\left(z^{8}\right),(30 \\
\sum_{n=1}^{\infty} \frac{(-1)^{n}}{n} \mathrm{e}^{-n z} & =\ln \left(1+\mathrm{e}^{-z}\right)^{-1} \simeq-\ln 2+\frac{z}{2}-\frac{z^{2}}{8}+\frac{z^{4}}{192}-\frac{z^{6}}{2880}+O\left(z^{8}\right)
\end{aligned}
$$

and the derivatives of (30) and (31) with respect to $z$ lead to (14) and (15) in the text.

\section{References}

[1] N. S. Manton, Nucl. Phys. B158 141 (1979).

[2] D. B. Fairlie, Phys. Lett. 82B (1979) 97.

[3] N. V. Krasnikov, Phys. Lett. 273B (1991) 731, H. Hatanaka, T. Inami and C.S. Lim, Mod. Phys. Lett. A13 (1998) 2601, G. R. Dvali, S. Randjbar-Daemi and R. Tabbash, Phys. Rev. D65 (2002) 064021, N. Arkani-Hamed, A. G. Cohen and H. Georgi, Phys. Lett. B513 (2001) 232, I. Antiniadis, K. Benakli and M. Quiros, New J. Phys. 3, (2001),20.

[4] Y. Hosotani, Phys. Lett. 126B (1983) 309, Ann. Phys. (N.Y.) 190, 233 (1989). 
[5] K. Takenaga, Phys. Rev. D64 (2001) 066001, Phys. Rev. D66 (2002) 085009, N. Haba and Y. Shimizu, Phys. Rev. D67 (2003) 095001, C. Csaki, C. Grojean, H. Murayama, Phys. Rev. D67 (2003) 085012 I. Gogoladze, Y. Mimura, S. Nandi and K. Tobe, Phys. Lett. B575 (2003) 66, C. Csaki, C. Grojean, H. Murayama, L. Pilo and J. Terning, Phys. Rev. D69 (2004) 055006, K. Choi, N. Haba, K. S. Jeong, K. Okumura, Y. Shimizu and M. Yamaguchi, JHEP 0402 (20037) 04, Y. Hosotani, S. Noda and K. Takenaga, Phys. Rev. D69 (2004) 125014, Phys. Lett. B607 (2005) 276 , N. Haba, K. Takenaga and T. Yamashita, Phys. Rev. D71 (2005) 025006, K. Oda and A. Weiler, Phys. Lett. B606 (2005) 408, K. Agashe, R. Contino and A. Pomarol, Nucl. Phys. B719 (2005) 165, Y. Hosotani and M. Mabe, Phys. Lett. B615 (2005) 257, Y. Hosotani, S. Noda, Y. Sakamura and S. Shimasaki, hep-ph/0601241.

[6] A. T. Davies and A. McLachlan, Phys. Lett. 200B (1988) 305, Nucl. Phys. B317 237 (1989), J. E. Hetrick and C. L. Ho, Phys. Rev. D40 (1989) 4085, A. Higuchi and L. Parker, Phys. Rev. D37 (1988) 2853, C. L. Ho and Y. Hosotani, Nucl. Phys. B345 445 (1990), A. McLachlan, Nucl. Phys. B338 188 (1990), K. Takenaga, Phys. Lett. 425B (1998) 114, Phys. Rev. D58 (1998) 026004.

[7] N. Haba, S. Matsumoto, N. Okada and T. Yamashita, JHEP 0602 (2006) 073.

[8] A. Masiero, C. A. Scrucca, M. Serone and L. Silvestrini, Phys. Rev. Lett. 87251601 (2001).

[9] S. Coleman and E. Weinberg, Phys. Rev. D7 (1973) 1888.

[10] N. Haba, K. Takenaga and T. Yamashita, Phys. Lett. B615 (2005) 247.

[11] G. Cacciapaglia, C. Csáki and S.C. Park, JHEP 0603 (2006) 099.

[12] K. Takenaga, Phys. Lett. B570 (2003) 244.

[13] N. Haba, K. Takenaga and T. Yamashita, Phys. Lett. B605 (2005) 355

[14] C.A.Scrucca, M.Serone and L. Silvestrini, Nucl. Phys. B669 128 (1903), G. Martinelli, M. Salvatori and C.A.Scrucca and L. Silvestrini, JHEP 0510 (2005) 037.

[15] G. Panico, M. Serone and A. Wulzer, .Nucl. Phys. B739 (2006) 186.

[16] M. Kubo, C. S. Lim and H. Yamashita, Mod. Phys. Lett. A17 (2002) 2249, L. J. Hall, Y. Nomura and D. R. Smith, Nucl. Phys. B639 (2002) 307, G. Burdman and Y. Nomura, Nucl. Phys. B656 (2002) 3.

[17] N. Maru and K. Takenaga, in preparation.

[18] N. Maru and K. Takenaga, Phys. Rev. D72 (2005) 046003. 
[19] A. Delgado, A. Pomarol and M. Quiros, Phys. Rev. D60 (1999) 095008.

[20] N. Haba, M. Harada, Y. Hosotani and Y. Kawamura, Nucl. Phys. B657 (2003) 169.

[21] N. Haba, Y. Hosotani, Y. Kawamura and T. Yamashita, Phys. Rev. D70 (2004) 01510.

[22] G. Panico and M. Serone, JHEP 0505 (2005) 024.

[23] L. Dolan and R. Jackiw, Phys. Rev. D9 (1974) 3320. 